\title{
Perturbation of Syndapin/PACSIN Impairs Synaptic Vesicle Recycling Evoked by Intense Stimulation
}

\author{
Fredrik Andersson, Joel Jakobsson, Peter Löw, Oleg Shupliakov, and Lennart Brodin \\ Department of Neuroscience, Karolinska Institutet, S-171 77 Stockholm, Sweden
}

Synaptic vesicle recycling has been proposed to depend on proteins which coordinate membrane and cytoskeletal dynamics. Here, we examine the role of the dynamin- and N-WASP (neural Wiskott-Aldrich syndrome protein)-binding protein syndapin/PACSIN at the lamprey reticulospinal synapse. We find that presynaptic microinjection of syndapin antibodies inhibits vesicle recycling evoked by intense ( $5 \mathrm{~Hz}$ or more), but not by light $(0.2 \mathrm{~Hz})$ stimulation. This contrasts with the inhibition at light stimulation induced by perturbation of amphiphysin (Shupliakov et al., 1997). Inhibition by syndapin antibodies was associated with massive accumulation of membranous cisternae and invaginations around release sites, but not of coated pits at the plasma membrane. Cisternae contained vesicle membrane, as shown by vesicle-associated membrane protein 2 (VAMP2)/synaptobrevin 2 immunolabeling. Similar effects were observed when syndapin was perturbed before onset of massive endocytosis induced by preceding intense stimulation. Selective perturbation of the Src homology 3 domain interactions of syndapin was sufficient to induce vesicle depletion and accumulation of cisternae. Our data show an involvement of syndapin in synaptic vesicle recycling evoked by intense stimulation. We propose that syndapin is required to stabilize the plasma membrane and/or facilitate bulk endocytosis at high release rates.

Key words: endocytosis; dynamin; lamprey; N-WASP; synaptic vesicle; syndapin

\section{Introduction}

Recycling of synaptic vesicles in nerve terminals can involve several distinct mechanisms (Murthy and De Camilli, 2003; Royle and Lagnado, 2003). A major mechanism is clathrin-mediated endocytosis during which clathrin-coated pits form at the plasma membrane in the periactive zone (Murthy and De Camilli, 2003; Royle and Lagnado, 2003). After invagination and fission, the coated vesicle sheds its coat before the vesicle is used in a new round of exocytosis. A second mechanism, bulk membrane retrieval, involves formation of deep plasma membrane invaginations, which are pinched off into vacuoles (Murthy and De Camilli, 2003; Royle and Lagnado, 2003). Synaptic vesicles are thought to form by clathrin-mediated budding from the vacuoles. Bulk membrane retrieval appears to be used preferentially during periods of intense release (Royle and Lagnado, 2003; Wu and $\mathrm{Wu}$, 2007). A third mechanism, kiss-and-run, implies that vesicles do not collapse into the plasma membrane, but recycle by closure of a fusion pore (Royle and Lagnado, 2003; Wu et al., 2007)

Actin is present at high concentrations in nerve terminals (Dillon and Goda, 2005). Although its role remains enigmatic, some observations have pointed to involvement in synaptic vesicle recycling. Actin filaments are primarily concentrated in the periactive zone in close proximity to endocytic intermediates,

\footnotetext{
Received April 18, 2007; revised Feb. 25, 2008; accepted Feb. 27, 2008.

This work was supported by Swedish Research Council Grants 11287 and 0 S 13473 to L.B., the Swedish Foundation for Strategic Research, The Human Frontier Science Program, and the Knut and Alice Wallenbergs Foundation. We thank Paul Kennedy for participation in the initial part of the work.

Correspondence should be addressed to Lennart Brodin, Department of Neuroscience, Karolinska Institutet, S-171 77 Stockholm, Sweden. E-mail: lennart.brodin@ki.se.

DOI:10.1523/JNEUROSCI.1754-07.2008

Copyright $\odot 2008$ Society for Neuroscience $\quad$ 0270-6474/08/283925-09\$15.00/0
}

and stimulation has been shown to promote growth of filament matrix (Dunaevsky and Connor, 2000; Gad et al., 2000; Shupliakov et al., 2002; Bloom et al., 2003; Sankaranarayanan et al., 2003; Richards et al., 2004; Bourne et al., 2006). At the molecular level, numerous interactions have been observed between endocytic proteins and actin regulators (Slepnev and De Camilli, 2000; Kessels and Qualmann, 2004). Defining the role of proteins mediating such interactions will be important to improve the understanding of synaptic vesicle recycling mechanisms.

Here, we focus on syndapin/PACSIN, a synaptically enriched protein of the F-BAR (FCH-BIN amphiphysin RVS)/PCH (Pombe Cdc15 homology) family (Itoh et al., 2005). It binds the actin-regulators neural Wiskott-Aldrich syndrome protein (NWASP) and Cobl in addition to dynamin and other proteins via a C-terminal Src homology 3 (SH3) domain (Qualmann et al., 1999; Modregger et al., 2000; Kessels and Qualmann, 2004; Kim et al., 2006; Tsujita et al., 2006; Ahuja et al., 2007). The $\mathrm{N}$-terminal consists of a F-BAR domain, which can bind to and deform phospholipid membranes (Peter et al., 2004; Itoh et al., 2005; Shimada et al., 2007). Previously, syndapin was implicated in synaptic vesicle endocytosis by the finding that stimulationinduced dephosphorylation of dynamin promotes formation of a syndapin-dynamin complex (Anggono et al., 2006). To examine the role of syndapin in vesicle recycling in the living synapse, we performed perturbation experiments in the lamprey giant axon (Brodin and Shupliakov, 2006). We find that presynaptic microinjection of syndapin antibodies or Fab fragments disrupts synaptic vesicle recycling activated by intense stimulation. Unlike compounds which perturb amphiphysin interactions (Shupliakov et al., 1997) the syndapin reagents had no detectable effect on vesicle recycling under conditions of low-frequency stimulation. 
The inhibition of recycling seen at intense stimulation was associated with massive build-up of cisternal structures that contained VAMP2/synaptobrevin 2.

\section{Materials and Methods}

Cloning and affinity chromatography. Lamprey (Lampetra fluviatilis) cDNA was made from purified brain mRNA using an oligo-DT primer with Thermoscript (Invitrogen, Carlsbad, CA). Using PCR and degenerated primers a 477-nucleotide-long fragment was amplified. This fragment was subcloned into the pCR2.1-topoisomerase (TOPO) vector using the TOPO-TA cloning kit (Invitrogen) and sequenced. The fragment (which showed a high degree of similarity to mammalian syndapin) was used to screen a cDNA library (Gad et al., 2000). Several clones were isolated and sequenced. Sequences were aligned using the ClustalW software (European Bioinformatics Institute, Cambridge, UK).

Full-length lamprey syndapin (442 aa; GenBank accession number ABS57012) and the SH3 domain of lamprey syndapin (amino acids 387442 ) were produced as glutathione $S$-transferase (GST) fusion proteins in bacteria using the pGEX-6P-2 vector and purified according to the manufacturers instruction (GE Healthcare, Little Chalfont, UK). Pull-down experiments with lamprey CNS lysate was performed as described previously (Evergren et al., 2004b). Binding assays were analyzed by SDSPAGE in parallel with Coomassie Blue staining and Western blotting according to standard procedures (Ringstad et al., 1999).

Antibodies. Recombinant full-length lamprey syndapin was used to immunize a rabbit. Affinity-purified antibodies were obtained using an $\mathrm{N}$-hydroxysuccinimide Hi-Trap column (GE Healthcare) with the antigen covalently linked. Fab fragments to the $\mathrm{SH} 3$ domain of lamprey syndapin were produced by first purifying the IgG with Protein A from rabbit serum. The IgG were cleaved using immobilized papain (Pierce Biotechnology, Rockford, IL), and the Fab fragments were then affinity purified against the $\mathrm{SH} 3$ domain of lamprey syndapin. Control injections were performed with protein A-purified rabbit IgG (Sigma). Antibodies to lamprey N-WASP were made by immunizing a rabbit with the VCA (verprolin homology-cofilin homology-acidic) domain fused with GST, followed by purification using Protein A Hi-Trap columns (GE Healthcare). Antibodies to rat vesicle-associated membrane protein 2 (VAMP2) will be described (J. Jakobsson, F. Andersson, O. Kjaerulff, F. Håkansson, P. Löw, O. Shupliakov, and L. Brodin, unpublished data). Dynamin was detected with antibodies to the GTPase domain of lamprey dynamin. The specificity of these antibodies in lamprey has been documented in previous studies (Evergren et al., 2004a).

Immunocytochemistry. Lampreys were anesthetized with MS-222 (tricaine methanesulphonate) and truncated before the spinal cords were dissected in ice-cold oxygenated Ringer's solution (Gad et al., 1998). The preembedding immunogold experiments were performed as described (Evergren et al., 2004a). Briefly, specimens were fixed in 3\% paraformaldehyde with $0.1 \%$ glutaraldehyde in $0.1 \mathrm{M}$ phosphate buffer. To expose the interior of axons the specimen was cut in longitudinal sections with a Vibratome. Microinjected specimens (see below) were in addition cut transversally at the injection site. Sections were blocked using $1 \%$ human serum albumin (HSA) in Tris-phosphate buffered saline, $\mathrm{pH}$ 7.4, and incubated for $16 \mathrm{~h}$ at $4^{\circ} \mathrm{C}$ with the primary anti-VAMP 2 antibody diluted in Tween 20 in PBS with 1\% HSA and secondary antibodies (5 nm gold; GE Healthcare). The sections were postfixed in 3\% glutaraldehyde in 0.1 $\mathrm{M}$ cacodylic buffer for $1 \mathrm{~h}$ and in $1 \%$ osmium tetroxide for $1 \mathrm{~h}$. After en bloc staining with uranyl acetate, sections were embedded in Durcupan ACM (Fluka, San Quentin Fallavier, France). Serial ultrathin or 250-nmthick sections were cut with a diamond knife, counterstained with uranylacetete and lead citrate and examined in a Tecnai 12 electron microscope (FEI, Ekerö, Sweden).

Microinjection. The isolated spinal cord was placed in a recording chamber with Ringer's solution maintained at $8^{\circ} \mathrm{C}$. The antibodies and Fab fragments were conjugated with Alexa fluorophores $(488,546,649$ or 680; Invitrogen) and diluted in $250 \mathrm{~mm} \mathrm{~K}^{+}$acetate and $10 \mathrm{~mm}$ HEPES, $\mathrm{pH}$ 7.4. Alexa labeled actin (488) (Invitrogen; $1.5 \mathrm{mg} / \mathrm{ml}$ ) was mixed with syndapin IgG or control IgG $(2.5 \mathrm{mg} / \mathrm{ml})$ in G-buffer $(2 \mathrm{~mm}$ Tris, $0.2 \mathrm{~mm}$ $\mathrm{Mg}^{2+}$ ATP (adenosine tri phosphate) and $0.2 \mathrm{~mm} \mathrm{CaCl}_{2}, \mathrm{pH}$ 8.0). Injec-

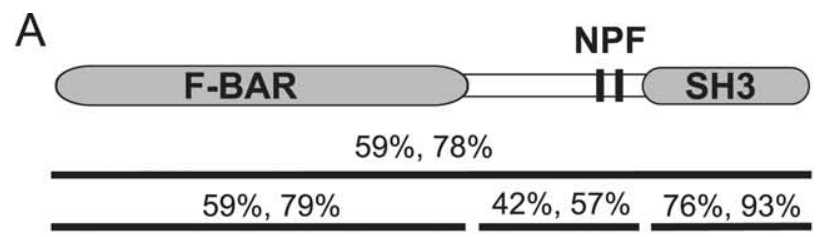

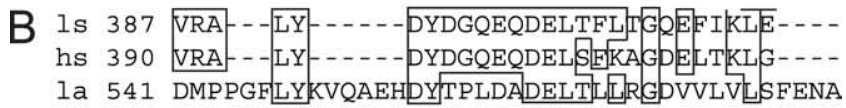
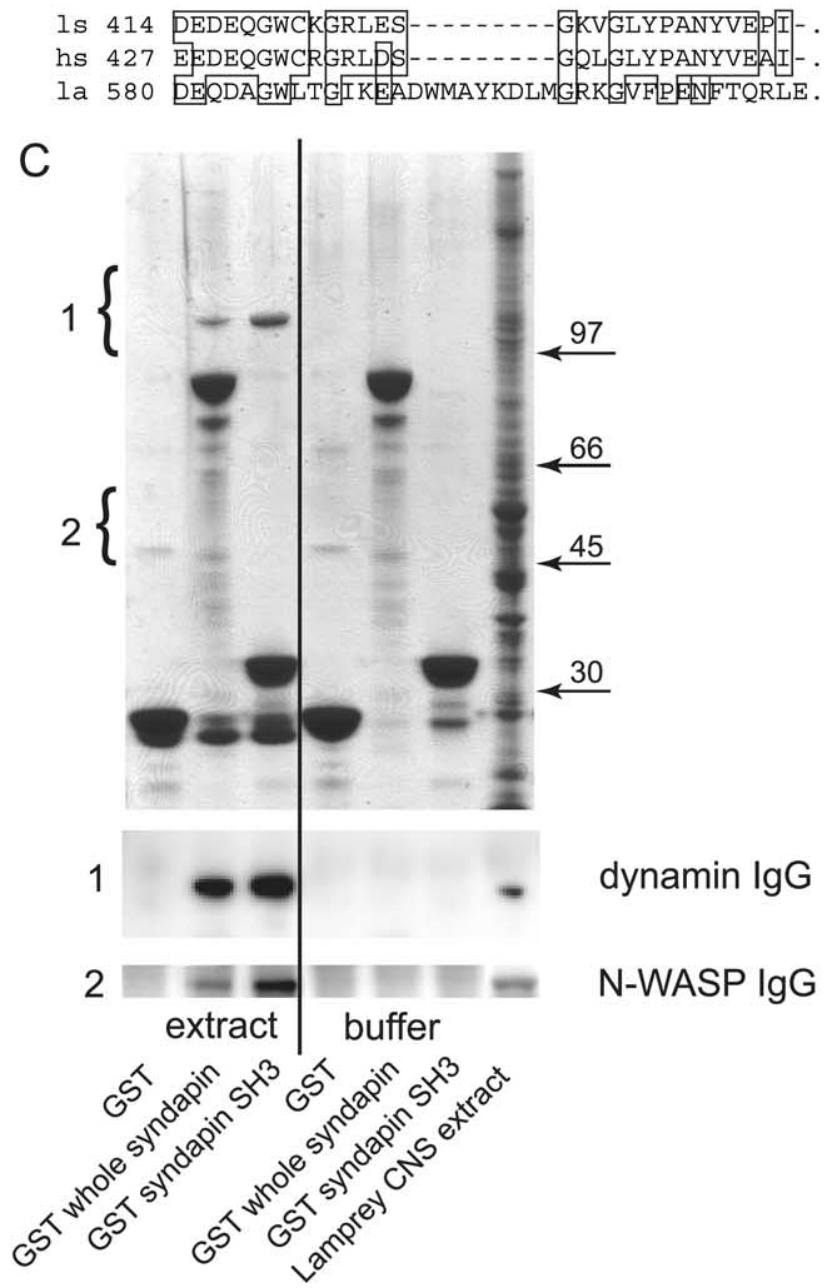

Figure 1. Characterization of lamprey syndapin. $\boldsymbol{A}$, Domain structure of the lamprey syndapin ortholog with the sequence identity and similarity to human syndapin 1 indicated (F-BAR domain, amino acids 1-326; SH3 domain, amino acids 387-442). $\boldsymbol{B}$, Alignment of SH3 domains from lamprey syndapin (Is), human syndapin (hs), and lamprey amphiphysin (la). C, Pull-down with GST fusion proteins of syndapin (full-length lamprey syndapin and isolated SH3 domain, respectively) from lamprey CNS extract. Top, Coomassie staining; bottom, immunoblot with antibodies against dynamin and N-WASP, respectively. Arrows indicate molecular weight markers $(n=3)$.

tions were monitored with a CCD detector (Roper Scientific, Trenton, NJ). Confocal images of living axons were obtained with a Nikon (Tokyo, Japan) Eclipse C1 system mounted on the same microscope stand as the CCD using a $40 \times / 0.8$ numerical aperture objective. Fluorescence intensities were measured with EZ-C1 software. In the actin imaging experiments, specimens were illuminated only at the beginning and end of the stimulation period to avoid fading.

Specimens were fixed by filling the chamber with fixative containing $2 \%$ tannic acid and $3 \%$ glutaraldehyde. The spinal cord was transected 1-2 $\mathrm{mm}$ from the injection site with a Vibratome to permit diffusion of 
A

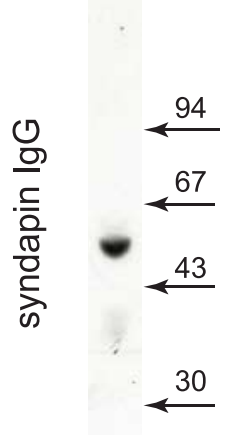

B

$$
\text { syndapin } \lg G \quad \text { VAMP } \lg G
$$

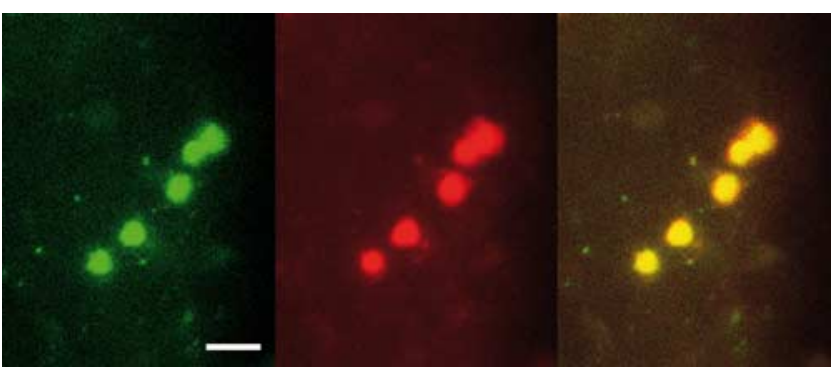

$0.2 \mathrm{~Hz}$, and antibodies were injected with pressure pulses. The fluorescence intensity in the region of synaptic contact was monitored with a CCD detector. In the experiments with $50 \mathrm{~Hz}$ stimulation (not physiological; see above), an axon was first microinjected with syndapin antibodies or inactive control antibodies. It was then reimpaled with a microelectrode containing $3 \mathrm{M} \mathrm{KCl}$ (to permit reliable activation at 50 $\mathrm{Hz}$ ) and a synaptically connected cell was thereafter identified. After recording the synaptic response at a rate of $0.2 \mathrm{~Hz}, 50 \mathrm{~Hz}$ stimulation was applied for $10 \mathrm{~min}$, followed by $0.2 \mathrm{~Hz}$ to record the recovery of the EPSP.

\section{Results}

Syndapin antibodies bind at release sites but do not disrupt synaptic function at low-frequency stimulation

As a first step toward analyzing syndapin function in the lamprey model, we isolated

tannic acid into axons. After 30 min the specimen was moved to $3 \%$ glutaraldehyde solution for $3 \mathrm{~h}$ or overnight, and thereafter postfixed and embedded as described above.

Morphometric analysis. All measurements were performed in serially sectioned synapses with a single active zone (Gustafsson et al., 2002). Synaptic vesicles were counted in the center section, coated pits were counted as the average from at least five sections around the center. Synaptic vesicles and coated pits were normalized to the length of the active zone. The length of active zones was measured using the NIH ImageJ software. To quantify cisternal membrane in synaptic regions, a mesh with squares of $200 \times 200 \mathrm{~nm}$ was placed over the synapse (center section). The percentage of squares containing membranous structures (with a longest diameter of $>100 \mathrm{~nm}$ to exclude synaptic vesicles) was counted in a rectangular area extending laterally one half active zone length (from the edges of the active zone) and into the axon one active zone length. Statistical analysis was performed using either GraphPad (San Diego, CA) Prism 3.0 or Excel (Microsoft, Redmond, WA) software. Student's $t$ test and Mann-Whitney test were used as appropriate.

Stimulation conditions. The stimulation conditions used have been described in previous studies. To suppress synaptic activity specimens were maintained unstimulated in "low $\mathrm{Ca}^{2+}$ Ringer" $\left(0.1 \mathrm{~mm} \mathrm{Ca}^{2+}\right.$ and $\left.4 \mathrm{~mm} \mathrm{Mg}^{2+}\right)$. At this condition signs of synaptic activity, including coated pits at the plasma membrane and synaptic vesicles scattered outside vesicle clusters, are not seen (Shupliakov et al., 1997; Ringstad et al., 1999). Stimulation at 0.2 and $5 \mathrm{~Hz}$ was applied via a suction electrode placed at the spinal cord surface. A rate of $0.2 \mathrm{~Hz}$ (30 min) was used to obtain a "minimal" level of activity (Shupliakov et al., 1997). A rate of 5 $\mathrm{Hz}(30 \mathrm{~min})$ was used to induce a high level of activity. At this rate the size of synaptic vesicle clusters (in control axons) is not reduced, suggesting that the capacity for vesicle recycling is not exceeded. To examine the recovery after $5 \mathrm{~Hz}$ stimulation the specimens were left in Ringer solution for $10 \mathrm{~min}$ and then incubated in low $\mathrm{Ca}^{2+}$ Ringer for $5 \mathrm{~min}$ before fixation. Stimulation at 20 or $50 \mathrm{~Hz}$, at which the capacity for recycling is exceeded, was performed with an intracellular microelectrode containing $3 \mathrm{M} \mathrm{KCl}$. It should be noted that, although reticulospinal neurons can fire in vivo at rates $>20 \mathrm{~Hz}$ (Zelenin, 2005), prolonged high-frequency firing (i.e., $30 \mathrm{~min}$ ) is unlikely to occur physiologically. In the experiments with dissociation between exocytosis and endocytosis, axons were stimulated at $20 \mathrm{~Hz}$ for $30 \mathrm{~min}\left(\right.$ at $\left.8^{\circ} \mathrm{C}\right)$ to induce depletion of synaptic vesicle clusters (Gad et al., 1998). At the end of the stimulation period, the specimen was rapidly cooled to $1^{\circ} \mathrm{C}$ by adding cold Ringer's solution. Microinjections were performed at this temperature and thereafter the temperature was maintained at $1-2^{\circ} \mathrm{C}$ for $30 \mathrm{~min}$, and then it was raised back to $8^{\circ} \mathrm{C}$ over a period of $40 \mathrm{~min}$.

Electrophysiology. The electrophysiological experiments were performed as described previously (Shupliakov et al., 1997; Gad et al., 2000). Briefly, after impaling a postsynaptic neuron a synaptically connected axon was impaled with an antibody-filled pipette. EPSPs were evoked at a syndapin orthologue from Lampetra fluviatilis CNS cDNA (Fig. $1 A$, similarity and identity to human syndapin 1 indicated). Like mammalian syndapin 1 it contains two NPF motifs. The SH3 domain (Fig. $1 B$ ) is highly homologous with that of human syndapin 1, but distinct from that of amphiphysin. Pull-downs with the full-length protein identified dynamin as the main binding partner in CNS (Fig. 1C, top, Coomassie staining). Binding with $\mathrm{N}$-WASP was verified by immunoblotting (Fig. $1 C$, bottom). The isolated SH3 domain of lamprey syndapin also bound dynamin and N-WASP (Fig. 1C).

Polyclonal antibodies raised against full-length lamprey syndapin reacted with a single $50 \mathrm{kDa}$ band in immunoblot on CNS extract (Fig. 2A). To examine whether the protein is accumulated at release sites syndapin antibodies were labeled with Alexa 488. After microinjection into the living reticulospinal axon the antibodies accumulated at release sites in a pattern overlapping with vesicle clusters marked by coinjected Alexa 546-labeled VAMP2 antibodies (Fig. 2B).

We next performed microinjection experiments designed to perturb syndapin function. To test whether synaptic transmission continues after microinjection of antibodies into the axon EPSPs were evoked at $0.2 \mathrm{~Hz}$. As shown in Figure $3 A$, the syndapin antibodies did not block synaptic transmission. Five min after the antibodies had reached the synaptic region, the mean EPSP amplitude was $96 \pm 21 \%$ of the preinjection value (mean \pm $\mathrm{SD} ; n=4$ synaptic connections in four animals).

Electron microscopy was used to examine synapses in axons stimulated at $0.2 \mathrm{~Hz}$ for $30 \mathrm{~min}$. No evident changes of the synaptic morphology were observed. Large vesicle clusters were present (Fig. 3B) with a number of synaptic vesicles (206.1 \pm 65.7 , mean $\pm \mathrm{SD}, n=16$ synapses from two different animals), which did not differ significantly from that in stimulated (adjacent) uninjected axons (Fig. 3C) (247.6 \pm 62.5 vesicles, mean $\pm \mathrm{SD}, n=13$ synapses from two different animals, $p>0.05)$. The number of clathrin-coated pits $(0.18 \pm 0.16$, mean $\pm \mathrm{SD}, n=13$ synapses from two different animals) neither differed from that in stimulated uninjected axons $(0.39 \pm 0.48$, mean $\pm \mathrm{SD}, n=13$ synapses from two different animals, $p>0.05)$. Antibodies to syndapin thus interact with syndapin at synaptic release sites, but this does not induce detectable impairment of synaptic structure or function at low-frequency stimulation. 


\section{Syndapin antibodies disrupt vesicle} recycling during intense stimulation

In the next series of experiments, we examined the involvement of syndapin in vesicle recycling under conditions of more intense action potential stimulation. We chose a rate of $5 \mathrm{~Hz}$ (30 min). Stimulation at this rate does not in itself induce vesicle depletion suggesting that endocytosis keeps pace with exocytosis (Shupliakov et al., 1997). In initial control experiments, we confirmed that the synaptic structure was largely maintained at this condition (supplemental Fig. 1 $A$, available at www. jneurosci.org as supplemental material). In addition, using preembedding immunogold labeling, we found that small VAMP2-immunopositive cisternae occurred in low numbers in the periactive zone (supplemental Fig. $1 B$, available at www.jneurosci.org as supplemental material). These cisternae may correspond to vesicle recycling intermediates or membrane infoldings evoked by intense action potential stimulation (Takei et al., 1996).

In axons microinjected with syndapin antibodies and then stimulated at $5 \mathrm{~Hz}$ for $30 \mathrm{~min}$ the synaptic structure was strikingly altered. Vesicle clusters were reduced in size and numerous large cisternae occupied the synaptic regions (Figs. 4, 5). Analysis of injected axons cut in serial semithin $(250 \mathrm{~nm})$ (Fig. 4) or ultrathin sections (60 nm) (Fig. 5) showed that every synapse contained numerous cisternae. Two observations suggested that the cisternae were linked with synaptic vesicle recycling. First, they were immunopositive to VAMP2 (Figs. 4, 5). Second, coated pits occurred on their surface (Fig. $5 B, C$, supplemental Fig. 2, available at www.jneurosci.org as supplemental material). Analysis of cisternae in serial sections showed that they were often interconnected in networks. In some cases cisternal networks could be traced to the site of connection with the plasma membrane (Figs. $4,5 D, E$, supplemental Fig. $3 A$, available at www.jneurosci.org as supplemental material). Such connections could have broad or narrow necks. In many cases, networks could not be traced in their entirety.

To quantify the effect of syndapin antibodies on endocytosis, we performed morphometric analysis. The number of synaptic vesicles was significantly decreased by syndapin antibodies (Fig. $6 A$ ). The decrease was paralleled by an increase in the amount of cisternae (Fig. $6 B$ ) and of coated pits associated with cisternae (Fig. 6C). The ultrastructural changes remained after a $15 \mathrm{~min}$ resting period. Notably, the number of coated pits on the plasma membrane proper (i.e., membrane apposed to another cell) was not altered by syndapin antibodies (Fig. 6D). This observation, along with the results in Figure 3 indicates that syndapin antibodies has little direct effect on clathrin-mediated endocytosis at the plasma membrane proper.

We also compared axons stimulated at $5 \mathrm{~Hz}$ with those stimulated at $0.2 \mathrm{~Hz}$ (in both cases with $2.6 \mathrm{mM} \mathrm{Ca}^{2+}$ ). In syndapin antibody-injected axons stimulated at $5 \mathrm{~Hz}$ the number of synaptic vesicles was significantly lower $(69.2 \pm 62.8$ vesicles mean \pm $\mathrm{SD}, n=5)$ than in axons stimulated at $0.2 \mathrm{~Hz}(206.1 \pm 65.7$ vesicles, $n=16, p<0.01)$. The amount of membranous cisternae was significantly higher at $5 \mathrm{~Hz}(34.9 \pm 23.4 \%, n=6)$ than at 0.2 $\mathrm{Hz}(10.1 \pm 7.2 \%$ mean $\pm \mathrm{SD}, n=12, p<0.01)$

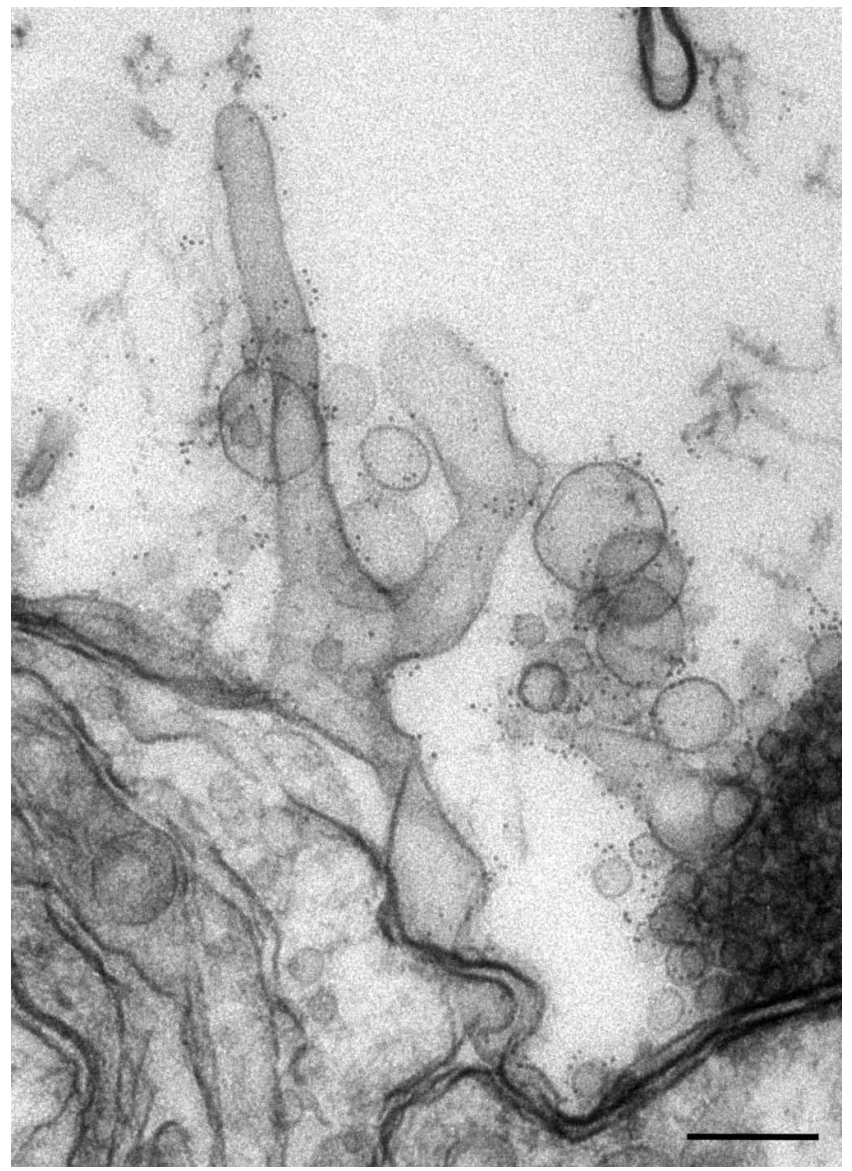

Figure 4. VAMP2-containing cisternae in syndapin antibody-injected axons stimulated at 5 $\mathrm{Hz}$. Micrograph of a synapse in a 250-nm-thick section of an axon microinjected with syndapin antibodies, stimulated at $5 \mathrm{~Hz}$ for $30 \mathrm{~min}$ and then maintained at rest for $15 \mathrm{~min}$. The axon was cut open (Evergren et al., 2004a) and labeled with VAMP2 antibodies from the cytoplasmic side. Note the gold particles associated with membrane cisternae and synaptic vesicles. Scale bar, 0.2 $\mu \mathrm{m}$. 
A

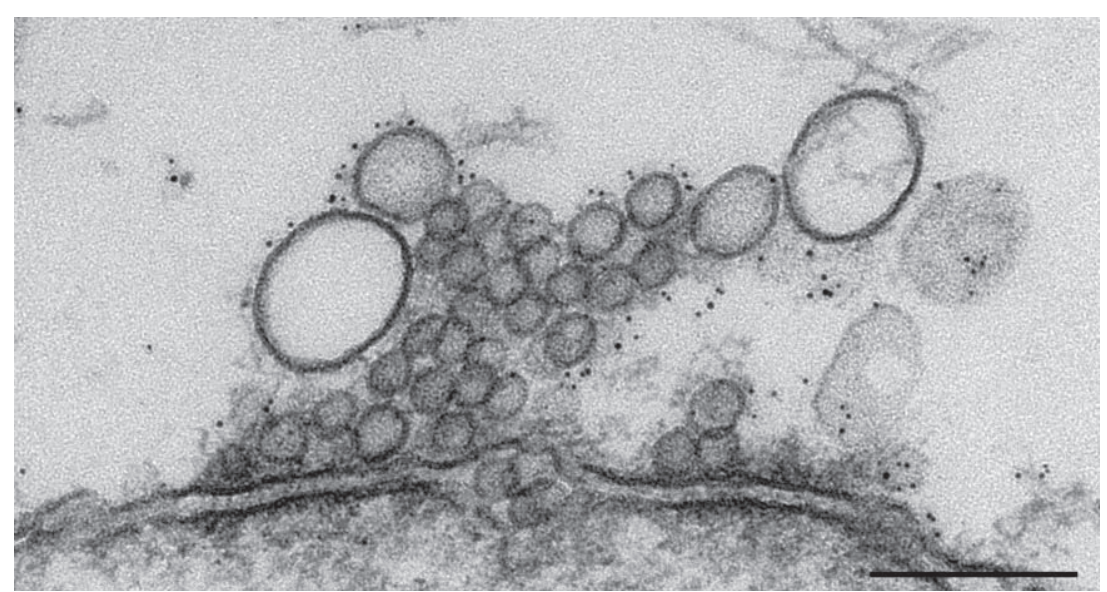

B
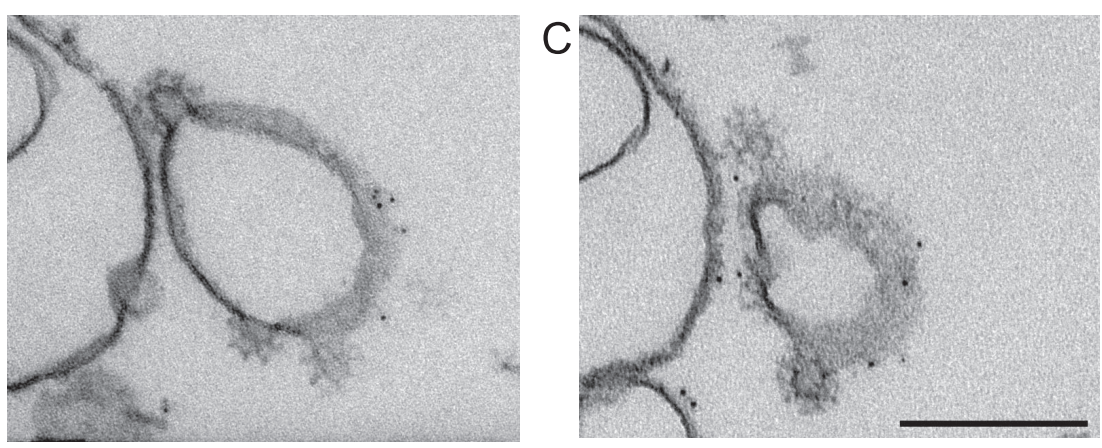

D

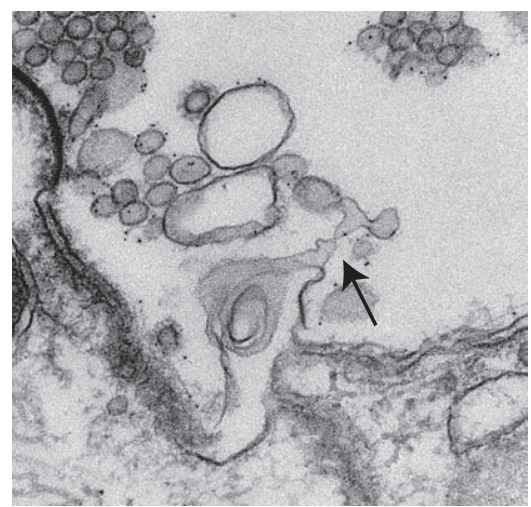

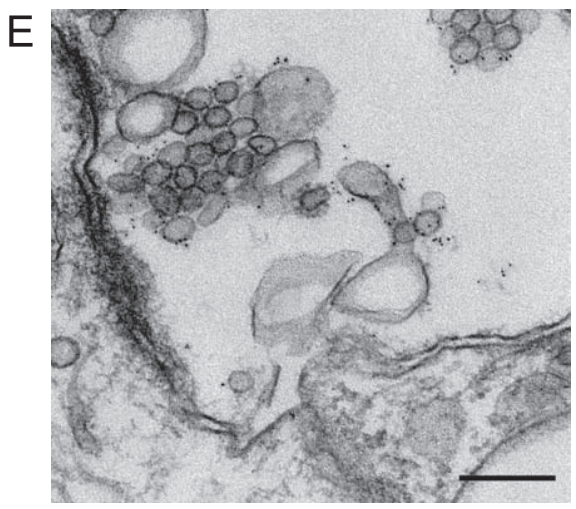

Figure 5. VAMP2-containing cisternae in syndapin antibody-injected axons stimulated at $5 \mathrm{~Hz}$. $\boldsymbol{A}$, Electron micrograph of an ultrathin section cut through the active zone in an axon labeled with VAMP 2 antibodies after stimulation at $5 \mathrm{~Hz}$ for $30 \mathrm{~min}$ and 15 min of rest. $\boldsymbol{B}, \boldsymbol{C}$, Adjacent sections through a cisternal structure with clathrin-coated pits located near a synapse in a similarly treated axon. $\boldsymbol{D}, \boldsymbol{E}$, Adjacent ultrathin sections including the periactive zone of a synapse in a similarly treated axon. Note the continuity between the cisternae and the plasma membrane (arrow). Scale bars, $0.2 \mu \mathrm{m}$.

Accumulation of cisternae after a single round of endocytosis To further investigate possible targets for the syndapin antibodies, we designed experiments to study their effect on endocytosis in the absence of ongoing exocytosis (Gad et al., 1998). Stimulation at $20 \mathrm{~Hz}$ was applied for $30 \mathrm{~min}$ to incorporate vesicle membrane into the plasma membrane (Fig. $7 A, B$ ). The specimen was then rapidly cooled from $8^{\circ} \mathrm{C}$ to $1^{\circ} \mathrm{C}$ to inhibit endocytosis and antibodies were microinjected at this temperature. Subsequent induction of endocytosis (by returning to $8^{\circ} \mathrm{C}$ ) led to full recovery of the synaptic morphology in control IgG-injected axons (Fig. 7C).

In synapses exposed to syndapin antibodies vesicle clusters failed to recover (number of synaptic vesicles, $64.8 \pm 17.5$, mean $\pm \mathrm{SD}, n=7$ synapses; recovered control, $185.2 \pm 47.6, n=$
8 synapses; $p<0.01, t$ test; similar results were obtained in a second independent experiment) (data not shown). Values were also compared with a separate experiment in which an axon was stimulated at $20 \mathrm{~Hz}$ for $30 \mathrm{~min}$ and then fixed directly after cooling to $1^{\circ} \mathrm{C}(104.60 \pm 36.42$ synaptic vesicles, $n=7$ synapses; $p>0.05$ compared with syndapin injected synapses and $p<0.01$ compared with recovered synapses, ANOVA, Bonferroni's post hoc test). Numerous cisternae occurred in syndapin antibody-injected synapses (area occupied by cisternae, $59.6 \pm 17.6 \%, n=7$ synapses; recovered control, $7.0 \pm 6.0 \%, n=$ 8 synapses; $p<0.001, t$ test). The cisternae were similar to those described above with regard to connections with other cisternae and the plasma membrane (Fig. 7E,F). Cisternae with coated pits were occasionally observed in antibody-injected synapses (Fig. 7G) (0.32 $\pm 0.17, n=7$ synapses), but none were detected in recovered control synapses $(n=7)$. A small number of coated pits occurred at the plasma membrane of injected (0.29 \pm $0.21, n=7$ synapses $)$ and control ( $0.15 \pm$ $0.16, n=7$ synapses; $p>0.05, t$ test $)$ axons. Similar results were obtained in a second independent experiment (data not shown). Thus, cisternae can be trapped by syndapin antibodies after only a single round of endocytosis.

\section{Enhanced depression of synaptic transmission}

To test whether the changes in synaptic morphology could be correlated with a decrease of synaptic transmission, we measured EPSPs before and after a period of intense stimulation ( $\mathrm{Gad}$ et al., 2000). In these experiments, we used a different protocol, which was designed to facilitate detection of possible EPSP changes $(50 \mathrm{~Hz}$ stimulation for $10 \mathrm{~min})$. The amplitude of EPSPs evoked by syndapin antibodyinjected axons showed an impaired recovery after stimulation (Fig. 6E, filled bars). In the case of control antibody-injected axons, the amplitude of EPSPs recovered normally (Fig. 6E, open bars) (Gad et al., 2000). Together with the EM data, these findings support an involvement of syndapin in the control of vesicle recycling during intense stimulation.

\section{Effects of perturbing syndapin on dynamin and actin at synapses}

Syndapin may play a role in recruiting dynamin (Anggono et al., 2006). Because release sites in the reticulospinal axon are not bounded by plasma membrane, we asked whether the effect of syndapin antibodies could be correlated with a loss of dynamin from release sites. Dynamin was monitored by coinjection of Alexa 488-conjugated dynamin antibodies and Alexa 546conjugated VAMP2 antibodies (to mark release sites). A preced- 

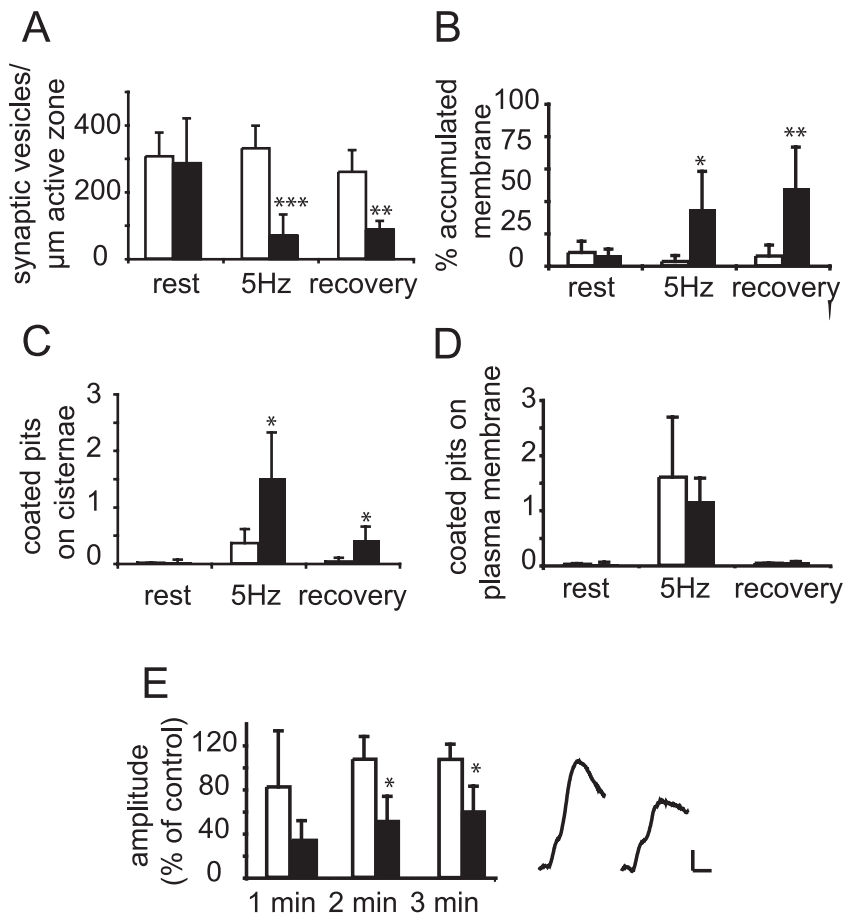

Figure 6. Quantitative analysis of the effects of syndapin antibody microinjection. $\boldsymbol{A}-\boldsymbol{D}$, Data from experiments with sustained $5 \mathrm{~Hz}$ stimulation, including values from axons maintained at rest, axons fixed directly after stimulation ( $5 \mathrm{~Hz}, 30 \mathrm{~min}$ ), and axons permitted to recover for $15 \mathrm{~min}$ after stimulation ( $5 \mathrm{~Hz}, 30 \mathrm{~min}$ ). Values for one representative experiment are shown. Similar results were obtained in at least three independent experiments (i.e., different animals). Open bars, Synapses in uninjected control axons; filled bars, synapses in adjacent axons injected with syndapin antibodies. $\boldsymbol{A}$, Number of synaptic vesicles per center section normalized to the length of the active zone. $\boldsymbol{B}$, Cisternal membrane structures in the synaptic region. The percentage of the synaptic area occupied by membranous structures larger than 100 $\mathrm{nm}$ (longest diameter) was measured. C, Number of coated pits located on cisternae. D, Coated pits on the plasma membrane proper (i. e., on regions of plasma membrane apposing the plasma membrane of another cell). Results are derived from five to six synapses in each group (mean \pm SD). $\boldsymbol{E}$, Recovery of reticulospinal EPSPs after intense stimulation. The EPSP amplitude was measured before and after $10 \mathrm{~min}$ of $50 \mathrm{~Hz}$ stimulation. The bars represent the averaged EPSP amplitude (recorded at $0.2 \mathrm{~Hz}$ ) during the first, second, and third minute after cessation of $50 \mathrm{~Hz}$ stimulation, normalized to the amplitude recorded before stimulation. Open bars, Axons injected with inactive control antibodies (mean \pm SD, $n=6$ synaptic connections in 6 animals). Filled bars, Axons injected with syndapin antibodies ( $n=6$, synaptic connections in 6 animals; $t$ test). Sample traces show averages before (left trace) and during the second min after (right trace) cessation of $50 \mathrm{~Hz}$ stimulation. Calibration: $5 \mathrm{~ms}, 0.5 \mathrm{mV} .{ }^{*} p<0.05$; ${ }^{* *} p<0.01$; ${ }^{* * *} p<0.001$.

ing injection of Alexa 680-conjugated syndapin antibodies followed by $30 \mathrm{~min}$ of $5 \mathrm{~Hz}$ stimulation did not alter the amount of dynamin accumulated at release sites (supplemental Fig. 4, available at www.jneurosci.org as supplemental material). Although this observation does not exclude that syndapin acts locally within the synapse to recruit dynamin, it indicates that the restriction of dynamin to release sites does not depend on syndapin.

To examine potential influences on actin in the periactive zone, we used Alexa-labeled actin (Bourne et al., 2006). Accumulation of actin fluorescence at the periactive zone was observed after coinjection of actin with either syndapin antibodies or with control antibodies (supplemental Fig. 5, available at www. jneurosci.org as supplemental material). However, the intensity of actin fluorescence was significantly reduced after $30 \mathrm{~min}$ of 5 $\mathrm{Hz}$ stimulation in axons coinjected with syndapin antibodies, but remained at a similar level in control-injected axons (supplemental Fig. 5, available at www.jneurosci.org as supplemental mate- rial). We conclude that actin turnover during synaptic activity is sensitive to disruption of syndapin function.

\section{Specific perturbation of the $\mathrm{SH} 3$ domain of syndapin impairs vesicle recycling}

We next examined the effect of perturbation of the $\mathrm{SH} 3$ domain of syndapin (Kessels and Qualmann, 2004). To achieve a selective perturbation, Fab fragments directed to the syndapin SH3 domain were generated. In pull-down experiments these Fab fragments inhibited binding of dynamin and N-WASP to syndapins SH3 domain (Fig. $8 \mathrm{~A}$ ) without affecting dynamin binding to the $\mathrm{SH} 3$ of amphiphysin used as control (data not shown). The Fab fragments were microinjected into reticulospinal axons followed by stimulation at $5 \mathrm{~Hz}$ for $30 \mathrm{~min}$. Electron microscopic analysis showed that synaptic vesicle clusters were significantly reduced in size $(60.5 \pm 22.8, n=5$ synapses; control, $164.5 \pm 19.2, n=5$ synapses; $p<0.001, t$ test) and numerous cisternae were present (Fig. $8 B, C)(44.3 \pm 14.8, n=5$ synapses; control, $6.1 \pm 7.8 \%, n=5$ synapses; $p<0.001, t$ test). Coated pits occurred on the cisternae (Fig. $8 C$ ) $(0.46 \pm 0.20, n=5$ synapses; control, $0.10 \pm 0.17 ; p<0.05$, $t$ test) and connections between cisternae and the plasma membrane were detected (Fig. $8 D$ ). The number of coated pits on the plasma membrane proper was similar in Fab fragment-injected (0.49 \pm $0.20, n=5$ synapses $)$ and control axons $(0.77 \pm 0.18, n=5 ; p>$ $0.05, t$ test). Similar results were obtained in a second independent experiment (data not shown).

\section{Discussion}

\section{Syndapin is required at high rates of synaptic activity}

We have used specific antibodies and Fab fragments in microinjection experiments to examine the role of syndapin at the lamprey reticulospinal synapse. We observed a stimulus-dependent loss of synaptic vesicles at a rate of $5 \mathrm{~Hz}$ along with appearance of numerous cisternae immunopositive for VAMP2. In contrast, no effect on synaptic ultrastructure was detected when the synapse was activated at $0.2 \mathrm{~Hz}$. This implies that syndapin function is selectively required for vesicle recycling at high rates of activity. Previously, Ferguson et al. (2007) showed that dynamin 1 is selectively needed during strong stimulation, and Mani et al. (2007) showed that this is the case also for the interaction between synaptojanin and endophilin. Thus, the vesicle recycling machinery appears not to be a constrained unit, but rather seems to be flexible, using different molecular components and interactions depending on the conditions of use.

\section{Syndapin and clathrin-mediated endocytosis}

Syndapin has been implicated in receptor-mediated endocytosis (Qualmann and Kelly, 2000; Kessels and Qualmann, 2004; PerezOtano et al., 2006), but its involvement in synaptic clathrinmediated endocytosis has not been tested previously. The reticulospinal axon has proved to be a useful model to study the latter mechanism. It contains isolated active zones surrounded by a periactive zone in which coated pits appear during stimulation (Brodin and Shupliakov, 2006). After perturbation of several endocytic proteins, the number of coated pits increases massively, in many cases $>10$ times (Shupliakov et al., 1997; Ringstad et al., 1999; Gad et al., 2000; Evergren et al., 2004b, 2007). Disruption of epsin ENTH function, which appears to inhibit coat nucleation, leads to a decrease in the number of coated pits (Jakobsson, H. Gad, Shupliakov, and Brodin, unpublished observation). After perturbation of syndapin the number of coated pits induced by stimulation $(0.2$ or $5 \mathrm{~Hz})$ did not differ from that in control axons, making it unlikely that syndapin plays a major role in 

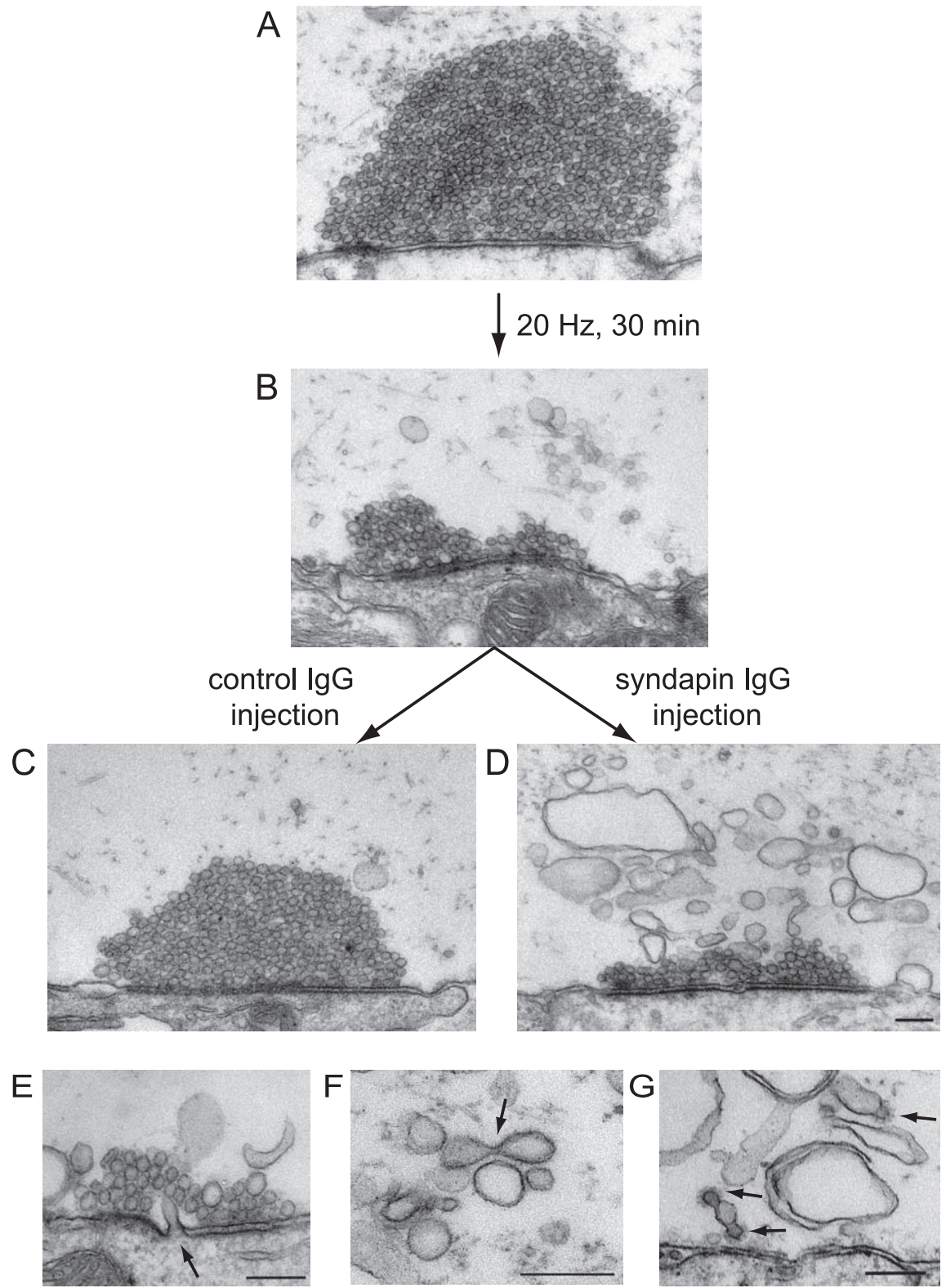

Figure 7. Accumulation of cisternae after a single round of endocytosis released in the presence of syndapin antibodies. $\boldsymbol{A}$, Unstimulated control synapse. $\boldsymbol{B}$, Synapse in an axon stimulated at $20 \mathrm{~Hz}$ for $30 \mathrm{~min}$ (at $8^{\circ}$ ) and then maintained at $1^{\circ} \mathrm{C}$ for 6 min until fixation. C, Synapse in an axon stimulated at $20 \mathrm{~Hz}$ for $30 \mathrm{~min}\left(\right.$ at $\left.8^{\circ}\right)$, then maintained at $1^{\circ} \mathrm{C}$ while inactive control antibodies were microinjected. The temperature was then raised to $8^{\circ}$ before fixation. The vesicle cluster has recovered. $D$, Synapse in an axon treated as in $\mathbf{C}$, but with microinjection of syndapin antibodies. The vesicle cluster has failed to recover and numerous cisternae have accumulated. $\boldsymbol{E}-\boldsymbol{G}$, Details from the experiment shown in $\boldsymbol{D}$. $\boldsymbol{E}$, Cisterna connected to the plasma membrane (arrow). $\boldsymbol{F}$, Thin connection between two cisternae (arrow). G, Coated pits (arrows) on cisternae. Scale bars, $0.2 \mu \mathrm{m}$.

clathrin-mediated synaptic vesicle endocytosis. Our preliminary immunogold observations indicate that syndapin occurs in the periactive zone, but is not associated with clathrin-coated pits (Andersson and Brodin, unpublished observations).

\section{Stabilization of the plasma membrane and bulk endocytosis}

After impairment of clathrin-mediated endocytosis the plasma membrane expands and can invaginate to appear as cisternae (Shupliakov et al., 1997; Ringstad et al., 1999; Gad et al., 2000; Evergren et al., 2004b, 2007). In the present study, however, cisternae formed in the absence of detectable effects on clathrinmediated endocytosis, suggesting that their occurrence cannot be merely explained by a compensatory expansion of the plasma membrane. To explain their occurrence two possibilities seem plausible. The first relates to the actin cytoskeleton in the periactive zone, which may act to stabilize the plasma membrane during sustained addition of vesicle membrane (Gad et al., 1998, 2000). Defects in actin function, caused by actin toxins or intersectin antibodies, have been found to induce plasma membrane invaginations (Shupliakov et al., 2002; Richards et al., 2004; Evergren et al., 2007) reminiscent of those observed here. The second explanation relates to bulk endocytosis which, however, is not yet a well characterized mechanism in central synapses. This process is thought to involve invagination of membrane portions, which are severed from the plasma membrane and then vesiculated by clathrin-mediated budding (Royle and Lagnado, 2003). Previous capacitance measurement studies in the calyx of Held synapse have confirmed that large membrane portions can indeed be severed from the plasma membrane ( $\mathrm{Wu}$ and $\mathrm{Wu}, 2007$ ). It is thus possible that cisternae seen here (or part of them) reflect intermediates of bulk endocytosis. The experiment with reversible cooling showed that cisternae occurred after a single round of endocytosis indicating that they, rather than resulting from a gradual invagination during sustained stimulation, could have reflected an endocytic process, such as bulk retrieval. The two mechanisms discussed above need not be mutually exclusive. On the contrary, plasma membrane stabilization and bulk retrieval, which appear to use common cytoskeletal components (Shupliakov et al., 2002; Holt et al., 2003; Richards et al., 2004), may operate jointly at high activity rates.

A role of syndapin in bulk endocytosis is consistent with its interactions with dynamin, Eps15 homology (EH domain) proteins, actin regulators and phospholipid membranes (Kessels and Qualmann, 2004; Anggono et al., 2006; Itoh and De Camilli, 2006). We speculate that syndapin is preferentially recruited to invaginated membrane portions, such as those formed by bulk retrieval. Recruitment may involve the F-BAR domain dimer, which has a shallow curvature (compared with BAR domains) and which may favor binding to cisternae and may also promote tubulation (Henne et al., 2007; Shimada et al., 2007). Interactions with $\mathrm{EH}$ domain proteins could contribute to recruitment (Braun et al., 2005) and/or promote EHD (EH domain-containing protein)-mediated tubulation. Calciumdependent dephosphorylation of dynamin specifically promotes its interaction with syndapin and this interaction has been proposed to activate endocytosis (Anggono et al., 2006; Evans and Cousin, 2007) (see also Kumashiro et al., 2005). It is possible that dynamin dephosporylation causes its recruitment to syndapin and/or promotes formation of a syndapin-dynamin complex that 
also contain N-WASP (Braun et al., 2005; Halbach et al., 2007) and possibly Cobl (Ahuja et al., 2007). Presumably, syndapin coordinates dynamin and actin functions, which may promote breakdown of cisternae. For instance, in cell expression studies dynamin was found to antagonize the plasma membrane-tubulating activity of F-BAR proteins, an activity that was enhanced by actin toxins (Itoh et al., 2005; Tsujita et al., 2006).

\section{References}

Ahuja R, Pinyol R, Reichenbach N, Custer L, Klingensmith J, Kessels MM, Qualmann B (2007) Cordon-bleu is an actin nucleation factor and controls neuronal morphology. Cell 131:337-350.

Anggono V, Smillie KJ, Graham ME, Valova VA, Cousin MA, Robinson PJ (2006) Syndapin I is the phosphorylation-regulated dynamin I partner in synaptic vesicle endocytosis. Nat Neurosci 9:752-760.

Bloom O, Evergren E, Tomilin N, Kjaerulff O, Löw P, Brodin L, Pieribone VA, Greengard P, Shupliakov O (2003) Colocalization of synapsin and actin during synaptic vesicle recycling. J Cell Biol 161:737-747.

Bourne J, Morgan JR, Pieribone VA (2006) Actin polymerization regulates clathrin coat maturation during early stages of synaptic vesicle recycling at lamprey synapses. J Comp Neurol 497:600-609.

Braun A, Pinyol R, Dahlhaus R, Koch D, Fonarev P, Grant BD, Kessels MM, Qualmann B (2005) EHD proteins associate with syndapin I and II and such interactions play a crucial role in endosomal recycling. Mol Biol Cell 16:3642-3658.

Brodin L, Shupliakov O (2006) Giant reticulospinal synapse in lamprey: molecular links between active and periactive zones. Cell Tissue Res 326:301-310.

Dillon C, Goda Y (2005) The actin cytoskeleton: integrating form and function at the synapse. Annu Rev Neurosci 28:25-55.

Dunaevsky A, Connor EA (2000) F-Actin is concentrated in nonrelease domains at frog neuromuscular junctions. J Neurosci 20:6007-6012.

Evans GJ, Cousin MA (2007) Activitydependent control of slow synaptic vesicle endocytosis by cyclin-dependent kinase 5. J Neurosci 27:401-411.

Evergren E, Tomilin N, Vasylieva E, Sergeeva V, Bloom O, Gad H, Capani F, Shupliakov O (2004a) A pre-embedding immunogold approach for detection of synaptic endocytic proteins in situ. J Neurosci Methods 135:169-174.

Evergren E, Marcucci M, Tomilin N, Löw P, Slepnev V, Andersson F, Gad H, Brodin L, De Camilli P, Shupliakov O (2004b) Amphiphysin is a component of clathrin coats formed during synaptic vesicle recycling at the lamprey giant synapse. Traffic 5:514-528.

Evergren E, Gad H, Walther K, Sundborger A, Tomilin N, Shupliakov O (2007) Intersectin is a negative regulator of dynamin recruitment to the synaptic endocytic zone in the central synapse. J Neurosci 27:379-390.

Ferguson SM, Brasnjo G, Hayashi M, Wolfel M, Collesi C, Giovedi S, Raimondi A, Gong LW, Ariel P, Paradise S, O’Toole E, Flavell R, Cremona O, Miesenbock G, Ryan TA, De Camilli P (2007) A selective activitydependent requirement for dynamin 1 in synaptic vesicle endocytosis. Science 316:570-574.

Gad H, Löw P, Zotova E, Brodin L, Shupliakov O (1998) Dissociation be-
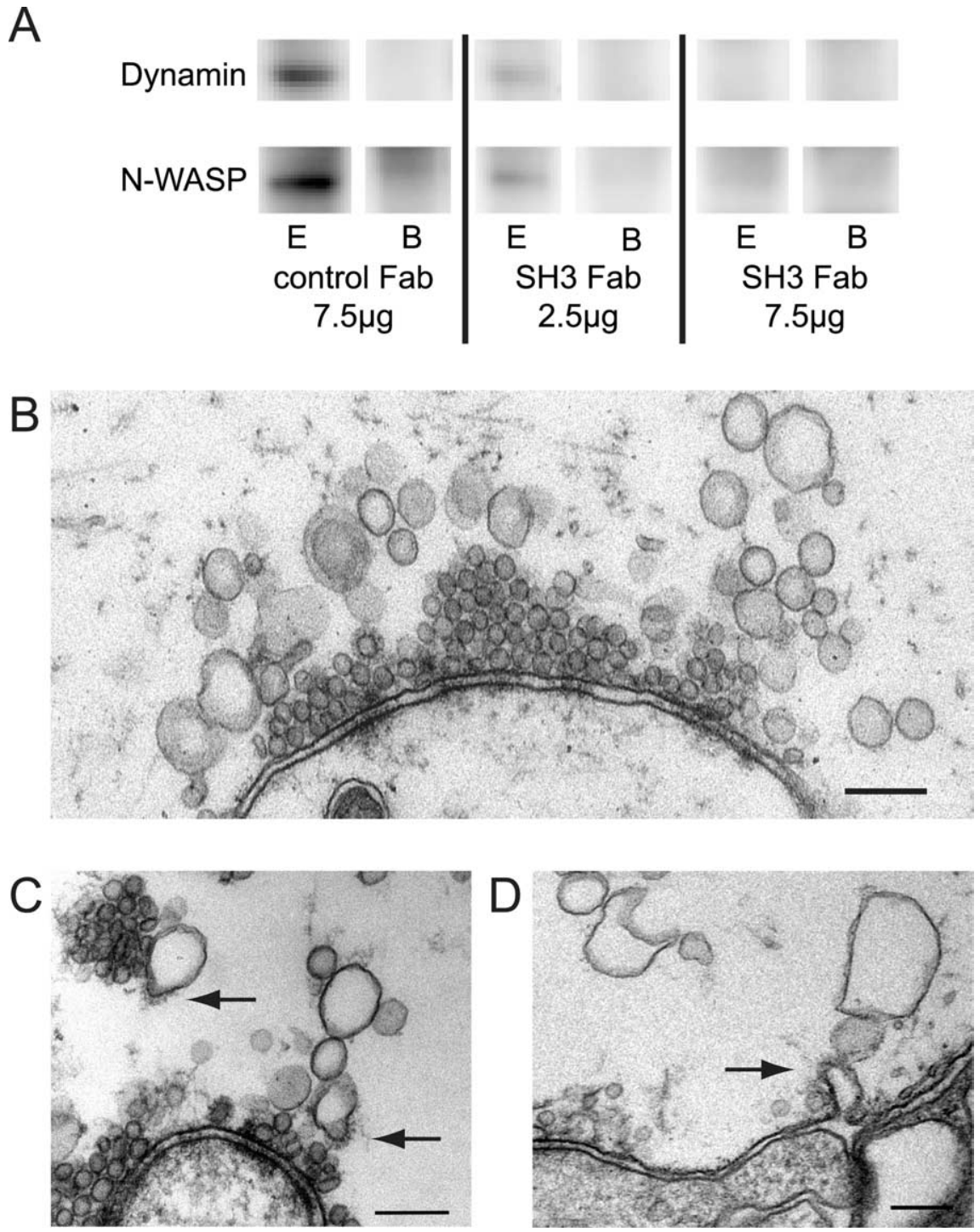

Figure 8. Fab fragments to the $\mathrm{SH} 3$ domain of syndapin cause vesicle depletion and accumulation of cisternae at stimulated B, Pull-down from lamprey CNS extract with the GST-SH3 domains of syndapin $(1 \mathrm{mg})$ analyzed by Western blot using connected with cisternae in a synapse treated as in $\boldsymbol{B}$. D, The margin of a synapse treated as in $\boldsymbol{B}$. Cisternae connected (arrow) with the plasma membrane occur in the periactive zone. Scale bars, $0.2 \mu \mathrm{m}$.

tween $\mathrm{Ca}^{2+}$-triggered synaptic vesicle exocytosis and clathrin-mediated endocytosis at a central synapse. Neuron 21:607-616.

Gad H, Ringstad N, Löw P, Kjaerulff O, Gustafsson J, Wenk M, Di Paolo G, Nemoto Y, Crun J, Ellisman MH, De Camilli P, Shupliakov O, Brodin L (2000) Fission and uncoating of synaptic clathrin-coated vesicles are perturbed by disruption of interactions with the $\mathrm{SH} 3$ domain of endophilin. Neuron 27:301-312.

Gustafsson JS, Birinyi A, Crum J, Ellisman M, Brodin L, Shupliakov O (2002) Ultrastructural organization of lamprey reticulospinal synapses in three dimensions. J Comp Neurol 450:167-182.

Halbach A, Morgelin M, Baumgarten M, Milbrandt M, Paulsson M, Plomann M (2007) PACSIN 1 forms tetramers via its N-terminal F-BAR domain. FEBS J 274:773-782.

Henne WM, Kent HM, Ford MG, Hegde BG, Daumke O, Butler PJ, Mittal R, Langen R, Evans PR, McMahon HT (2007) Structure and analysis of FCHo2 F-BAR domain: a dimerizing and membrane recruitment module that effects membrane curvature. Structure 15:839-852.

Holt M, Cooke A, Wu MM, Lagnado L (2003) Bulk membrane retrieval in the synaptic terminal of retinal bipolar cells. J Neurosci 23:1329-1339. 
Itoh T, De Camilli P (2006) BAR, F-BAR (EFC) and ENTH/ANTH domains in the regulation of membrane-cytosol interfaces and membrane curvature. Biochim Biophys Acta 1761:897-912.

Itoh T, Erdmann KS, Roux A, Habermann B, Werner H, De Camilli P (2005) Dynamin and the actin cytoskeleton cooperatively regulate plasma membrane invagination by BAR and F-BAR proteins. Dev Cell 9:791-804.

Kessels MM, Qualmann B (2004) The syndapin protein family: linking membrane trafficking with the cytoskeleton. J Cell Sci 117:3077-3086.

Kim SH, Choi HJ, Lee KW, Hong NH, Sung BH, Choi KY, Kim SM, Chang S, Eom SH, Song WK (2006) Interaction of SPIN90 with syndapin is implicated in clathrin-mediated endocytic pathway in fibroblasts. Genes Cells 11:1197-1211.

Kumashiro S, Lu YF, Tomizawa K, Matsushita M, Wei FY, Matsui H (2005) Regulation of synaptic vesicle recycling by calcineurin in different vesicle pools. Neurosci Res 51:435-443.

Mani M, Lee SY, Lucast L, Cremona O, Di Paolo G, De Camilli P, Ryan TA (2007) The dual phosphatase activity of synaptojanin 1 is required for both efficient synaptic vesicle endocytosis and reavailability at nerve terminals. Neuron 56:1004-1018.

Modregger J, Ritter B, Witter B, Paulsson M, Plomann M (2000) All three PACSIN isoforms bind to endocytic proteins and inhibit endocytosis. J Cell Sci 113 Pt 24:4511-4521.

Murthy VN, De Camilli P (2003) Cell biology of the presynaptic terminal. Annu Rev Neurosci 26:701-728.

Perez-Otano I, Lujan R, Tavalin SJ, Plomann M, Modregger J, Liu XB, Jones EG, Heinemann SF, Lo DC, Ehlers MD (2006) Endocytosis and synaptic removal of NR3A-containing NMDA receptors by PACSIN1/syndapin 1 . Nat Neurosci 9:611-621.

Peter BJ, Kent HM, Mills IG, Vallis Y, Butler PJ, Evans PR, McMahon HT (2004) BAR domains as sensors of membrane curvature: the amphiphysin BAR structure. Science 303:495-499.

Qualmann B, Kelly RB (2000) Syndapin isoforms participate in receptormediated endocytosis and actin organization. J Cell Biol 148:1047-1062.

Qualmann B, Roos J, DiGregorio PJ, Kelly RB (1999) Syndapin I, a synaptic dynamin-binding protein that associates with the neural Wiskott-Aldrich syndrome protein. Mol Biol Cell 10:501-513.

Richards DA, Rizzoli SO, Betz WJ (2004) Effects of wortmannin and latrunculin A on slow endocytosis at the frog neuromuscular junction. J Physiol (Lond) 557:77-91.
Ringstad N, Gad H, Löw P, Di Paolo G, Brodin L, Shupliakov O, De Camilli P (1999) Endophilin/SH3p4 is required for the transition from early to late stages in clathrin-mediated synaptic vesicle endocytosis [see comments]. Neuron 24:143-154.

Royle SJ, Lagnado L (2003) Endocytosis at the synaptic terminal. J Physiol (Lond) 553:345-355.

Sankaranarayanan S, Atluri PP, Ryan TA (2003) Actin has a molecular scaffolding, not propulsive, role in presynaptic function. Nat Neurosci 6:127-135.

Shimada A, Niwa H, Tsujita K, Suetsugu S, Nitta K, Hanawa-Suetsugu K, Akasaka R, Nishino Y, Toyama M, Chen L, Liu ZJ, Wang BC, Yamamoto M, Terada T, Miyazawa A, Tanaka A, Sugano S, Shirouzu M, Nagayama K, Takenawa T (2007) Curved EFC/F-BAR-domain dimers are joined end to end into a filament for membrane invagination in endocytosis. Cell 129:761-772.

Shupliakov O, Löw P, Grabs D, Gad H, Chen H, David C, Takei K, De Camilli $\mathrm{P}$, Brodin L (1997) Synaptic vesicle endocytosis impaired by disruption of dynamin-SH3 domain interactions. Science 276:259-263.

Shupliakov O, Bloom O, Gustafsson JS, Kjaerulff O, Low P, Tomilin N, Pieribone VA, Greengard P, Brodin L (2002) Impaired recycling of synaptic vesicles after acute perturbation of the presynaptic actin cytoskeleton. Proc Natl Acad Sci USA 99:14476-14481.

Slepnev VI, De Camilli P (2000) Accessory factors in clathrin-dependen synaptic vesicle endocytosis. Nat Rev Neurosci 1:161-172.

Takei K, Mundigl O, Daniell L, De Camilli P (1996) The synaptic vesicle cycle: a single vesicle budding step involving clathrin and dynamin. J Cell Biol 133:1237-1250.

Tsujita K, Suetsugu S, Sasaki N, Furutani M, Oikawa T, Takenawa T (2006) Coordination between the actin cytoskeleton and membrane deformation by a novel membrane tubulation domain of $\mathrm{PCH}$ proteins is involved in endocytosis. J Cell Biol 172:269-279.

Wu LG, Ryan TA, Lagnado L (2007) Modes of vesicle retrieval at ribbon synapses, calyx-type synapses, and small central synapses. J Neurosci 27:11793-11802

Wu W, Wu LG (2007) Rapid bulk endocytosis and its kinetics of fission pore closure at a central synapse. Proc Natl Acad Sci USA 104:10234-10239.

Zelenin PV (2005) Activity of individual reticulospinal neurons during different forms of locomotion in the lamprey. Eur J Neurosci 22:2271-2282. 